\title{
A Diffusion Tensor Imaging Study on the Auditory System and Tinnitus
}

\author{
Alessandro Crippa ${ }^{\mathrm{a}}$, Cris P. Lanting ${ }^{\mathrm{b}}$, Pim van Dijk ${ }^{\mathrm{b}, \mathrm{c}}$ and Jos B.T.M. Roerdink, ${ }^{*, \mathrm{a} c}$ \\ ${ }^{a}$ Johann Bernoulli Institute for Mathematics and Computer Science, University of Groningen, The Netherlands; \\ ${ }^{b}$ Department of Otorhinolaryngology, University Medical Center Groningen, University of Groningen, The Netherlands; \\ ${ }^{c} B C N$ Neuroimaging Center, University of Groningen, The Netherlands
}

\begin{abstract}
Tinnitus is an auditory percept in the absence of an external sound source. Mechanisms in the central nervous system are believed to be key in the pathophysiology of tinnitus. Diffusion tensor imaging (DTI) is an MR imaging technique that allows in vivo exploration of white matter tissue in the human brain. Using a probabilistic DTI approach, we determined the characteristics of fiber tracts from the inferior colliculus to the medial geniculate body up to the primary auditory cortex. We also investigated the connections between the auditory system and the amygdala, which may be involved in some forms of tinnitus. White matter tracts were characterized by three quantities: the mean fractional anisotropy, the weighted mean fractional anisotropy and the path strength. All these quantities are measures of the patency of white matter tracts. The most important finding is an increased patency of the white matter tracts between the auditory cortex and the amygdala in tinnitus patients as compared to healthy controls.
\end{abstract}

Keywords: Tinnitus, diffusion tensor imaging, connectivity patterns, lateralization.

\section{INTRODUCTION}

\subsection{Auditory System and Tinnitus}

The central auditory system starts at the auditory nerve (AN) which conveys action potentials in response to neurotransmitters released by the hair cells in the cochlea. The cochlear nucleus $(\mathrm{CN})$ is the first nucleus of the auditory system receiving information from the ipsilateral cochlea. A next step in the pathway is the superior olivary complex (SOC), in which input from the two cochlear nuclei converge. Neurons from the $\mathrm{CN}$ and SOC project to the inferior colliculus (IC) through axons that form the lateral lemniscus (LL). The next step is formed by connections between the inferior colliculus and the medial geniculate body (MGB) of the thalamus, a relay station of several types of information of which the auditory pathway is only one. Fibers leaving the MGB project to the primary auditory cortex (AC). For more detailed information we refer to Ehret and Romand [1]. In addition to this classical auditory pathway there are connections between the auditory system and the limbic system [2]. The limbic system is involved in motivation, mood and emotion [3], and consists of many subsystems, including the hippocampus, the amygloid complex, the cingulate gyrus and the prefrontal cortex [4]. Typical complaints attached to tinnitus such as anxiety, depression, and emotions such as fear indicate the association of the limbic system with tinnitus [5]. Cognitive therapies focus on the reduction of alteration of the emotional content of the percept of tinnitus by habituation

\footnotetext{
*Address correspondence to this author at the Johann Bernoulli Institute for Mathematics and Computer Science, University of Groningen, P.O. Box 407, 9700 AK Groningen, The Netherlands. Tel: +31-50-3633931; Fax: +31-50-3633800.; E-mail: j.b.t.m.roerdink@rug.nl
}

$[6,7]$. Changes in regional cerebral blood flow (rCBF) and blood oxygenation (BOLD) signal have been reported in the limbic system by several studies [8]. The connection between the auditory system and the limbic system may thus be of importance in the pathology of tinnitus.

In this paper we study the characteristics of white matter fiber tracts defining the (classical) auditory pathway, especially the pathways from the IC to the MGB up to the primary auditory cortex. We also investigate the connections between the auditory system and the limbic system, especially the amygdala (AM). Both pathways were studied using diffusion tensor imaging (DTI) methods. Additionally, we investigated possible differences in structural brain connectivity between subjects affected by tinnitus and healthy subjects. Since hemispheric differences have been reported in patients [9-11], also lateralization of DTI findings was investigated.

\subsection{Diffusion Tensor Imaging}

Diffusion tensor imaging (DTI) is a recently developed MR acquisition modality that enables the measurement of structural organization of tissues $[12,13]$. As a powerful and non-invasive technique for in vivo exploration of human tissues, DTI is widely used in various medical fields, especially in brain imaging $[14,15]$.

DTI is based on the diffusion properties of water molecules in white matter of the brain. The diffusion is limited by the fibrous nature of white matter: the wellorganized axon structure, axon membranes, neurofilaments and overall the myelin coating surrounding the neurons induce the displacement of water molecules to occur preferentially along the axon fibers rather than perpendicularly to them [16]. This anisotropic diffusion of water molecules can be measured by an MR scanner, allowing us to infer information on white matter connectivity. Two 
interesting features of DTI techniques are the ability to derive local information, such as the amount of anisotropy and the principal water diffusion direction in a single brain voxel [17], and the possibility to track fiber bundles from a selected brain area [18].

Although several imaging studies have examined the auditory system and tinnitus [10, 19, 20], only a few of them applied DTI techniques [21-23]. The main reason for the infrequent application of DTI in studies on the auditory system is the poor spatial resolution of DTI images. Partial volume effects lead to underestimation of diffusion anisotropy, and multiple nerve fiber tracts crossing a single brain voxel may disturb the fiber tracking algorithm. According to tracing studies on the macaque [24] using radioactive tracers, we know that the fibers of the auditory pathway intersect motor bundles (in the CN-SOC-IC path) and are close (within millimeter range) to the corticospinal tract (in the IC-MGB path) and do cross the internal capsule [25] when connecting the MGB with the AC.

Standard deterministic fiber tracking techniques only consider the main diffusion direction in each voxel, which is provided by the eigenvector corresponding to the largest eigenvalue of the diffusion tensor in the voxel. This technique is incapable of resolving voxels with multiple fiber directions that occur when two fibers cross each other within a voxel, but also has problems in the presence of gray matter voxels. For this reason, we used probabilistic tractography with multiple fiber orientations [26]; this technique has been shown to provide significant advantages in sensitivity when tracking non-dominant fiber populations and allowed us to track the auditory paths, which are impossible to detect with deterministic tractography. Although probabilistic tractography does not allow the visualization of the actual fiber bundles, it outputs a whole-brain probabilistic connectivity map for localizing white matter tracts. Using this method, we investigated the connections of the auditory pathways and the connections between the auditory system and the limbic system in a focused per-subject analysis. We also studied differences in tractography results of these paths in subjects with and without subjective tinnitus.

\subsection{Related Work on the Auditory System}

The auditory system and tinnitus have been studied using functional imaging techniques $[10,19,20]$. DTI methods have been used in a few studies on the auditory system, but in most cases only scalar values derived from DTI analysis, such as the fractional anisotropy (FA) index, describing the amount of anisotropy per voxel, were considered. For instance, Lee et al. [23] performed a study where the FA index in several areas of the brain was determined. Reduced FA values in tinnitus patients were found in the left frontal Arcuate Fasciculus and the right parietal Arcuate Fasciculus. However, the classical auditory pathway was not studied. Lutz et al. [22] were able to detect changes in FA maps in cortical and subcortical auditory regions, in relation with the age of the subjects (although the study did not concern tinnitus): elder subjects showed bigger FA values in IC and lower FA values in the auditory radiation and in the temporal gyri. Moreover, Lee et al. [27] showed differences in FA values between subjects with conductive hearing loss and subjects with profound sensorineural hearing loss, reporting that DTI findings of subjects with profound sensorineural hearing loss revealed neural damages in the auditory pathway. De Groof et al. [28] used DTI to study the auditory and vocal system in birds. They discerned a number of song control and auditory nuclei, and discriminated the tracts running from and to these nuclei. Using DTI fiber tracking techniques, Upadhyay et al. [29] studied the connectivity patterns within the primary auditory cortex.

Different methods are available for exploring differences in brain structure between two groups. Using voxel-based morphometry (VBM), brain data from different subjects are aligned to a reference volume so that voxel-wise statistics can be computed [30]. This method has been used for comparing diffusion tensor data $[31,32]$ and provides a whole-brain picture of structural differences; the major drawback of this technique is that the results could be strongly influenced by the quality of the alignment among the brain data and by the spatial smoothing usually applied to the data [33]. Tract-based spatial statistics [33] is another tool for comparing DTI data among subjects. This technique seems to be very robust to misalignment of brain data; however, it only allows comparisons of high-FA white matter voxels and is not able to analyze a specific fiber bundle per se.

\section{MATERIALS}

\subsection{Subjects}

DTI data were acquired from 25 subjects: 15 healthy subjects and 10 subjects with tinnitus. The group of healthy volunteers consisted of 12 men and 3 women; the age of these varied from 26 to 75 , with a mean of 46 and a standard deviation of 16. The subjects affected by tinnitus ( 9 men and 1 woman) had ages varying from 30 to 70 , with an average of 49 and a standard deviation of 12 . All the subjects were right-handed. Six of the patients suffered from bilateral tinnitus. Four suffered from tinnitus in the left ear only. Fig. (1A) shows the hearing levels of the patients at different octave frequencies; Fig. (1B) shows the severity of tinnitus as perceived by the patients during rest, according to a visual analogue scale (VAS) ranging from 1 to 10 . The frequency and loudness level of tinnitus were determined by a matching procedure. Frequency matching was performed with an external tone presented at the non-tinnitus ear (unilateral tinnitus) or at both ears (bilateral tinnitus) at a comfortable level. The loudness level was then determined by adjusting the level of this tone to match the tinnitus loudness. All subjects gave written informed consent for their participation in the study.

\subsection{Data Acquisition}

All the imaging experiments were performed on a $3 \mathrm{~T}$ Philips Intera MRI scanner. DTI was performed using a diffusion weighted spin-echo, echo-planar imaging technique. The DTI parameters were as follows: field of view $=240 \times 240 \mathrm{~mm}$; matrix size $=128 \times 128$; no. of slices $=50$; imaging resolution $=1.85 \times 1.85 \times 2 \mathrm{~mm}^{3} ; \mathrm{TR}=5485$ $\mathrm{ms} ; \mathrm{TE}=74 \mathrm{~ms}$. In total, 61 volumes were acquired per subject, one without diffusion weighting $\left(b=0 \mathrm{~s} / \mathrm{mm}^{2}\right)$ and 60 volumes with diffusion weighting $\left(b=800 \mathrm{~s} / \mathrm{mm}^{2}\right)$ along 60 noncollinear directions. To correct for susceptibility artefacts, two acquisitions were used: one with fat-shift 


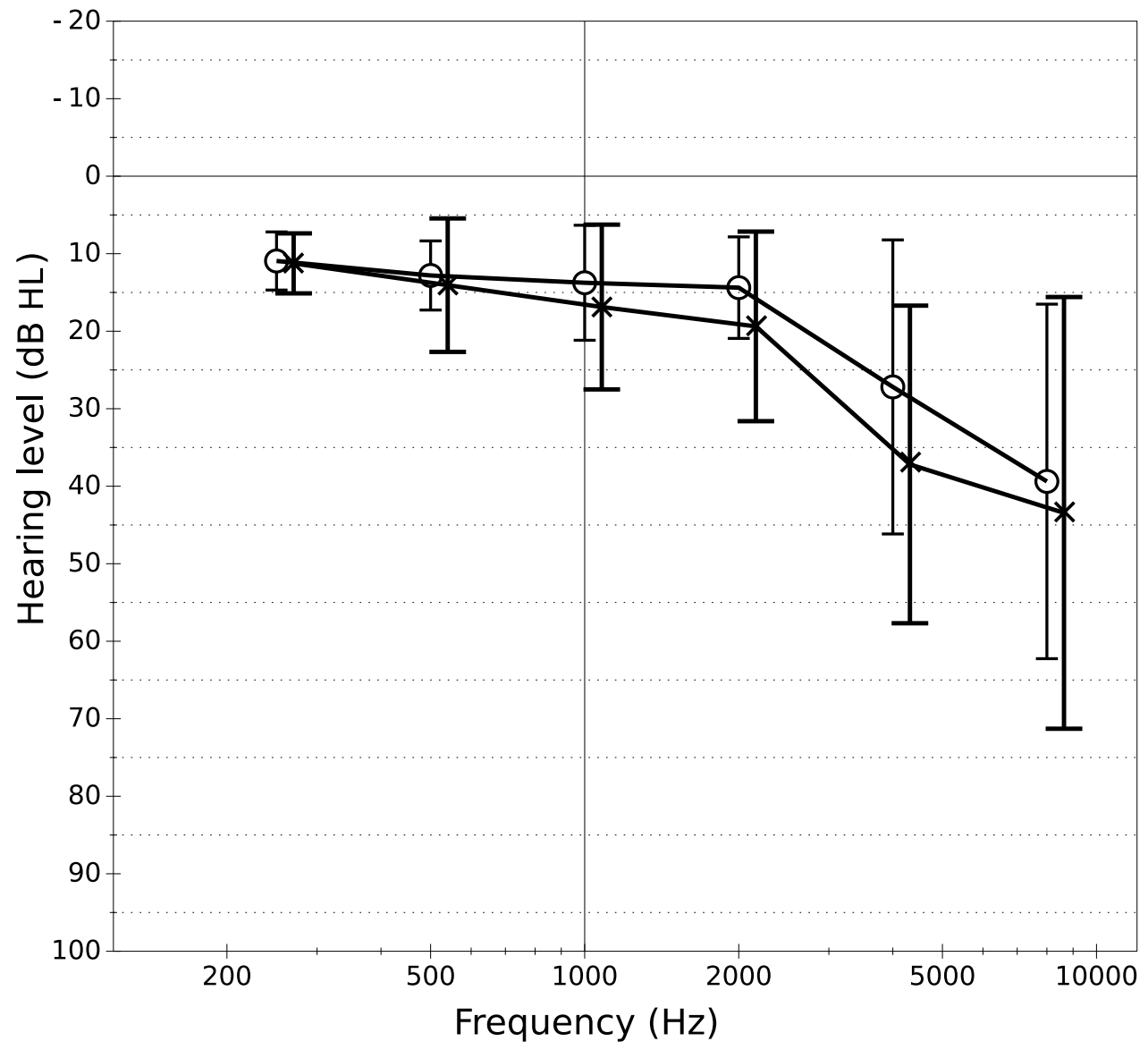

\begin{tabular}{l|cccccccccc} 
patient & 1 & 2 & 3 & 4 & 5 & 6 & 7 & 8 & 9 & 10 \\
\hline VAS & $\mathrm{X}$ & $\mathrm{X}$ & 1 & 5 & 4 & 4 & 7 & 10 & 3 & 7.5 \\
\hline freq $(\mathrm{kHz})$ & 0.75 & 8 & 9 & 4 & 4 & 9 & 6 & 0.75 & 9 & 11 \\
\hline loudness (dB SL) & 12 & 13 & 10 & 10 & 20 & 15 & 15 & 15 & 15 & $\mathrm{X}$
\end{tabular}

Fig. (1). A: Hearing levels (in $\mathrm{dB} \mathrm{HL}$ ) of the tinnitus patients at several frequencies. Circles represent the average levels for the right ear, crosses represent average levels for the left ear. The whiskers show standard deviations. $B$ : Severity (VAS), frequency and loudness level (in $\mathrm{dB}$ SL) of tinnitus perceived by the patients. An " $\mathrm{X}$ " is used when data were not available.

direction in the posterior direction (APP) and one in the anterior direction (APA).

An anatomical scan was acquired to serve as reference (T1-weighed fast-field echo scan, $\mathrm{TR}=25 \mathrm{~ms}, \mathrm{TE}=4.5 \mathrm{~ms}$, flip angle $=30^{\circ}$, imaging resolution $=0.94 \times 0.94 \times 1 \mathrm{~mm}^{3}$, matrix $=256 \times 256 \times 160$ slices $)$.

For the region of interest (ROI) selection, functional MRI data were acquired. These consisted of $2179 \mathrm{~ms}$ single shot T2 ${ }^{*}$-sensitive echo planar imaging (EPI) sequences, 41 slices, $2 \mathrm{~mm}$ thickness, TR $=10 \mathrm{~s}$, TE $=22 \mathrm{~ms}$, flip angle $30^{\circ}$, matrix $128 \times 128$, field of view $224 \mathrm{~mm}$, SENSE reduction factor $2.7,51$ acquisition per subject. The influence of acoustic scanner noise on fMRI data was reduced by using a sparse sampling strategy [34]. A TE of 22 ms was chosen for optimal SNR at areas close to air-tissue boundaries, like the temporal lobes, at the cost of a small SNR decrease in the BOLD signal (see e.g., the introduction of [35]).

Auditory stimuli were delivered by a MR-compatible electrodynamic system (MR Confon $\mathrm{GmbH}$ [36]). This system was driven by a PC equipped with a digital-analogue card (National Instruments 6052E) controlled by Labview 6.1 (National Instruments Corporation, Austin, TX). The auditory stimuli were generated off-line using Matlab ${ }^{\circledR}$ and consisted of temporally and spectrally modulated broadband "rippled" noise [37]. The stimuli had a frequency-range of $125-8000 \mathrm{~Hz}$ with a spectral modulation density of 1 cycle per octave, a temporal modulation frequency of 2 cycles per second and a modulation-amplitude of $80 \%$. 
Each of the three functional runs consisted of the acquisition of 61 volumes under 3 experimental conditions: (i) a condition in which bilateral rippled noise noise was presented at a level of $70 \mathrm{~dB}$ (SPL), (ii) a condition in which subjects were visually instructed to protrude their jaw, and (iii) a combination of these two conditions (jaw protrusion + sound stimuli). Each condition was presented 15 times per functional run.

\section{METHODS}

First we investigated the auditory connections and the connections between the auditory and the limbic system using standard deterministic DTI tracking provided by TrackVis [38]. This method was inadequate and failed to reveal any connections. Therefore we reverted to probabilistic tracking [39]. While standard DTI tracking computes a single main diffusion direction per voxel, probabilistic DTI tries to determine the most probable diffusion direction in each voxel by computing voxel-wise probability distributions of the main diffusion direction. The technique consists of sampling these distributions in each voxel to find the probabilities to reach the neighboring voxels, and repeating the process in the voxels reached. In this way it is possible to compute the probability to reach any voxel, starting from a certain voxel (or group of voxels).

\subsection{Preprocessing}

Before DTI analysis, preprocessing of raw data is necessary. This preprocessing step includes susceptibility correction, as well as eddy current and gradient table correction, depending on the scanner used for the acquisition. Susceptibility correction, which requires two DTI acquisitions with different fat-shift directions, was performed using MatLab, combined with the Statistical Parametric Mapping toolbox [40], the FieldMap toolbox [41], and the MatLab routines created by Andersson et al. [42]. Correction for eddy currents was performed with the software package FSL [39], a library of analysis tools for fMRI, MRI and DTI data. The gradient table modification was achieved using a MatLab toolbox created by Farrell et al. [43]. The last step before tracking is skull stripping (i.e., disregarding all non-brain data) and it was performed using BET [44].

\subsection{Region of Interest Selection}

We studied a number of brain areas involved in auditory processing and investigated how they are connected to each other. In particular, we determined the connectivity profiles between auditory cortex and inferior colliculus (AC-IC), auditory cortex and amygdala (AC-AM), inferior colliculus and amygdala (IC-AM) and all reverse connections. We disregarded the connections between inferior colliculus and cochlear nucleus (IC-CN), because a preliminary analysis showed that the motor fibers in the brain stem, running next to the acoustic fibers, have a strong influence on the results due to the poor spatial resolution of DTI. The selection of these ROIs (AC, IC, and AM) was done manually and for each individual patient, using the anatomical $\mathrm{T} 1$ scan as a primary reference. When possible (in 6 datasets), the location of the AC was checked by a comparison with BOLD activation maps (contrast: sound - baseline) provided by fMRI analysis. Location and size of the ROIs were checked by overlapping the drawn ROIs, normalized to the Montreal Neurological Institute (MNI-152) template brain [45], to the correspondent areas defined by the Juelich Histological Atlas, thresholded at 5\% [46, 47]; ACs showed an average overlap of $70 \%$, AMs showed an average overlap of $77 \%$.

Following Anwander et al. [48], each ROI selection included both the gray matter and a part of the white matter directly beneath it. This was done to avoid dispersion of samples already at the early stages of the tracking procedure, due to the low anisotropy of gray matter areas. In this way the first part of the axon bundles leaving from the concerned gray matter area was included in the ROI.

To prevent the tractography from finding connections passing through cortical regions or the cerebrospinal fluid (CSF), masks were defined where tracts could not enter. These were positioned in the CSF above the AC and along Reil's Insula, and served as barriers to avoid tracts passing through these regions. Nevertheless, the usage of these masks was not sufficient and after the probabilistic tracking an additional filtering was necessary, i.e., fiber tracts leading to the cerebellum or to the motor cortex were manually removed.

\subsection{Probabilistic Tractography}

The BEDPOST tool [49], which runs a Markov Chain Monte Carlo sampler, was used for building distributions of parameters describing the diffusion direction in each voxel. Probabilistic tractography was accomplished using the FMRIB Diffusion Toolkit [49]. For each voxel in a ROI, 5000 samples were taken from the distribution.

The probabilistic tractography outputs a connectivity map depicting the voxel-wise probability to reach any given voxel starting from a user-defined ROI, and it is defined as the percentage of samples leaving from the starting ROI that pass through that voxel. The connectivity map was then filtered so that only those paths connecting two different ROIs, a seed and a target ROI, were considered. An intrinsic problem of the sampling is that regions nearby the starting ROI are reached by a large number of samples, whereas the further a region is from the starting ROI, the fewer samples are able to reach it. This is a well-known and still unsolved problem. We addressed it by computing the connections between two ROIs in both directions: first starting from a region and reaching the other one, and then the other way around. Statistics on the paths, as described in the next section, were computed independently on each single path.

\subsection{Statistical Analysis}

Although the voxel-wise FA value is the conventional statistical measure used in DTI analysis, we considered various novel statistics to compare the two groups. For each path between a seed ROI and a target ROI (and vice versa) the following three statistical values were computed. First, the mean FA value of a path was determined as the mean of the FA values of all voxels in the path. Second, the weighted mean $F A$ value (wFA) of a path was determined, where the weighting was provided by the probability value of each voxel (i.e., the chance that a voxel is reached as determined by the probabilistic tracking). Voxels with a larger weight 
are more likely to represent the actual anatomical pathway. The third statistical value was the strength (S) of a path, defined as the percentage of the samples leaving from the starting ROI that were able to reach the target ROI. We consider the strength of a path as a key feature that describes the relevance of that path with respect to any other path connecting the starting ROI to other brain areas. Additionally, hemispheric differences were determined for all three statistical values (features) for each subject and path, using a lateralization index $L$ defined as:

$L_{\text {feature }}=2\left(\right.$ feature $_{\text {right }}-$ feature $\left._{\text {left }}\right) /\left(\right.$ feature $_{\text {right }}+$ feature $\left._{\text {left }}\right)$.

These statistical values were used to compare the two groups, resulting in a distribution of statistical values for each group and path. Since a chi-square test revealed that the distributions were not normally distributed, we used the nonparametric Kruskal-Wallis test in which the equality of the medians of the distributions is assessed to test for differences between control subjects and tinnitus patients.

\section{RESULTS}

The fiber tracts of the classical auditory system and of the connections between the auditory nuclei and the limbic system could be identified by the probabilistic tracking technique. These connections were consistent between the two directions of tracking. We also ascertained that standard deterministic DTI tracking is not able to reveal these connections. However, probabilistic tractography was not able to detect every path in a statistically significant way for every subject. The easiest path to track was the auditory path, which was detected in $50 \%$ (from AC to IC) and $35 \%$ (from IC to AC) of the subjects, respectively. The connection between IC and AM was found in $40 \%$ of the cases (50\% from AM to IC), and the path AC-AM (and vice versa) was found in $40 \%$ of the subjects. We did not find any relation between the cases where it was impossible to find a path and the type of subject (control or tinnitus patient). It appears that the difficulties in tracking the paths are due only to the low imaging resolution or to the low signal-to-noise ratio. No significant differences were found between the positions of the paths in the control group and the tinnitus group: all subjects showed the same connectivity pattern. This was true both for the connections between the auditory nuclei (AC and IC) and for the connections between the auditory nuclei and the amygdala.

The results of the statistical analysis of differences between tinnitus patients and control subjects are summarized in Table $\mathbf{1}$ and in Fig. (2). Statistics were computed on each single path connecting two different ROIs. Note that in this phase of the analysis, we consider the

Table 1. Significance Values (p-values) Determined by the Kruskal-Wallis Test of Differences Between Tinnitus Group and Control Group

(a): Differences in Path Indices Between the Groups for Each Hemisphere

\begin{tabular}{|c|c|c|c|c|c|c|}
\hline Left hem. & AC-IC & AC-AM & IC-AC & IC-AM & AM-AC & AM-IC \\
\hline \hline FA & 0.943 & 0.148 & 0.630 & 0.108 & 0.033 & 0.732 \\
\hline wFA & 0.936 & 0.624 & 0.261 & 0.935 & 0.739 & 0.262 \\
\hline S & 0.127 & $\mathbf{0 . 0 2 0}$ & 0.377 & $\mathbf{0 . 0 1 6}$ & AM-AC \\
\hline right hem. & AC-IC & AC-AM & IC-AC & IC-AM & 0.422 & 0.631 \\
\hline FA & 0.423 & 0.333 & 0.149 & 0.192 & 0.74 \\
\hline wFA & 0.521 & 0.106 & 0.423 & 0.199 & 0.109 & 0.135 \\
\hline S & $\mathbf{0 . 0 4 7}$ & $\mathbf{0 . 0 0 6}$ & 0.149 & 0.631 \\
\hline
\end{tabular}

(b): Differences in Path Lateralization Between the Groups. Significant p-values (0.05 Threshold) are Indicated in Bold

\begin{tabular}{|c|c|c|c|}
\hline & FA & wFA & S \\
\hline \hline AC-IC & 0.377 & 0.262 & 0.631 \\
\hline AC-AM & $\mathbf{0 . 0 1 0}$ & $\mathbf{0 . 0 3 7}$ & 0.261 \\
\hline IC-AC & 0.053 & $\mathbf{0 . 0 2 4}$ & 0.108 \\
\hline IC-AM & 0.628 & 0.628 & 0.333 \\
\hline AM-AC & 0.739 & 0.618 & 0.998 \\
\hline AM-IC & 0.423 & 0.994 & 0.631 \\
\hline
\end{tabular}



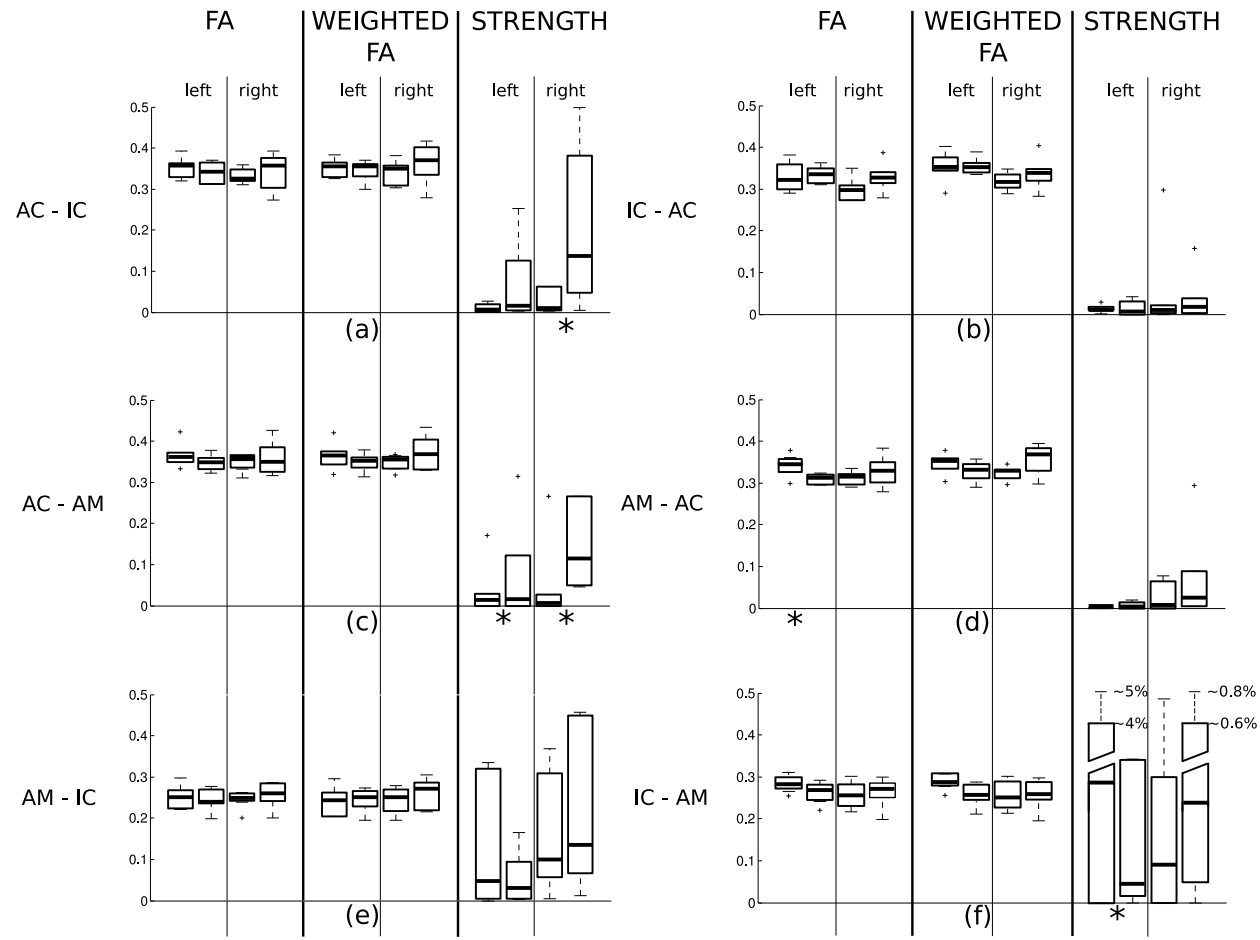

Fig. (2). Box \& whisker plots of the distributions of FA, weighted FA and strength in the paths of tinnitus patients and controls. For each pair of box and whiskers, the left data set corresponds to control subjects and the right one to tinnitus patients. Panel (a) shows the statistical results computed on the tract AC-IC; panel (b) of the tract IC-AC, panel (c) of the tract AC-AM, panel (d) of the track AM-AC, panel (e) of the tract AM-IC, panel (f) of the tract IC-AM. Paths where a statistically significant difference between tinnitus patients and controls was found (see Table 1(a)) are marked with an asterisk below the plot.

path from a ROI "A" to a ROI "B" different from the path from "B" to "A". Table 1a) shows the significance values (pvalues) determined by the Kruskal-Wallis test of differences between tinnitus patients and control subjects in fractional anisotropy (of the detected paths), weighted fractional anisotropy (of the detected paths) and strength of all the paths. The test detects a number of significant differences between the paths of the two groups. Statistically significant differences (threshold 0.05 ) can be noted in the strength of the AC-AM connection: it is higher in the tinnitus group, as seen from Fig. (2), both for the right and the left hemisphere. Other significant differences are present in the FA values of the path AM-AC in the left hemisphere and in the strengths of the path $\mathrm{AC}-\mathrm{IC}$ in the right hemisphere (stronger in the tinnitus group) and the path IC-AM in the left hemisphere (stronger in the control group).

Lateralization of FA, weighted FA and strength of the paths in the two groups is shown in Fig. (3). Table $\mathbf{1 b}$ shows the significance values ( $\mathrm{p}$-values determined using the Kruskal-Wallis test) of the differences in these indices between the two groups. Statistically relevant differences (threshold 0.05) can be noted in the lateralization of FA and of weighted FA in the path AC-AM, and in the lateralization of weighted FA in the path IC-AC. In both cases the tinnitus group shows a right lateralization whereas the control group shows a left lateralization, as can be seen from Fig. (3).

So far, statistics on the paths were computed independently on each single path between ROIs. For the sole purpose of visualizing the auditory paths as found by the probabilistic tractography, we used the intersection of the two paths $(\mathrm{A} \rightarrow \mathrm{B}, \mathrm{B} \rightarrow \mathrm{A})$ between any pair $(\mathrm{A}, \mathrm{B})$ of ROIs to define the location of a connection. For this purpose we normalized all the paths to the MNI template brain [45] using FLIRT [50], and averaged the per-subject results to obtain a group-wise connectivity map. The result is shown in Fig. (4). The paths in Fig. (4A) show connections between $\mathrm{AC}$ and IC. These paths also involve the MGB through which they pass. Note that the MGB was not used as a ROI. In Fig. (4B) we show the connections between the amygdala and the inferior colliculus, overlapped with the connections between the amygdala and the auditory cortex. The two paths follow the same route from the AM to the MGB, where they split into two separate paths, one to the IC and one to the AC.

\section{DISCUSSION}

In this study we extended the application of DTI of the brain to the study of auditory pathways in tinnitus patients and controls. We considered DTI tracks that connect the inferior colliculus, the auditory cortex and the amygdala and vice versa. In other words, we only considered tracks that connected these preselected seed and target ROIs. Obviously, such an approach will not identify new connections. Rather, it allows for the quantification of known connections in the brain. 
The first interesting result is the ability to track the classical auditory pathway. The tracks that connect the AC and the IC all pass through the MGB. Thus, although the MGB was not preselected as a region of interest, these tracks follow the expected pathway of the classical auditory system. Hence, as in a recent validation study performed in the macaque [51], DTI identifies known neuronal tracks in the brain.
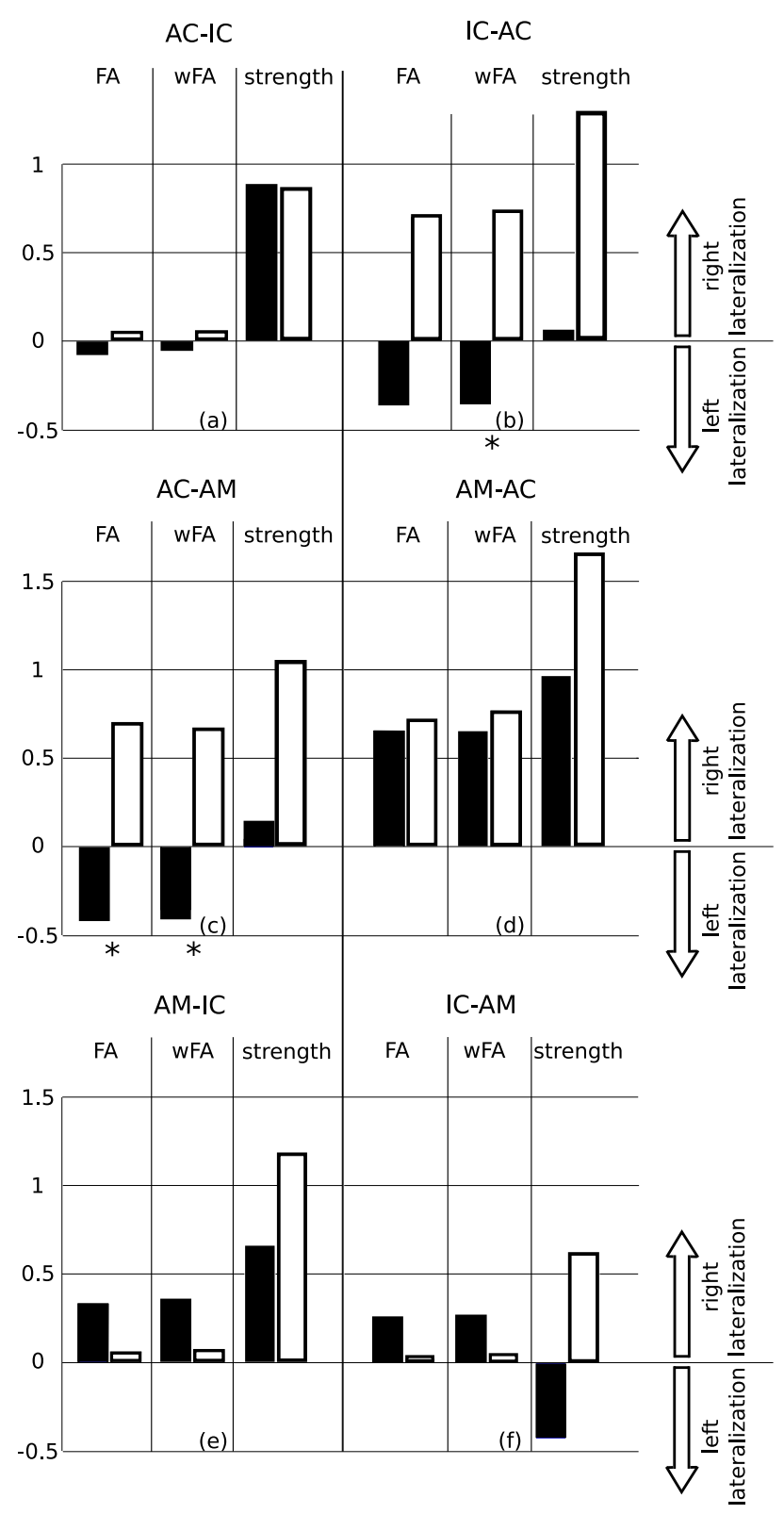

Fig. (3). Lateralization of the paths in the two groups. The black bars represent lateralization in the control group, the white bars represent lateralization in the tinnitus group. Positive values mean right lateralization, and negative values mean left lateralization (the range of the lateralization is $[-2,2]$ ). The Kruskal-Wallis test (see Table 1b) found significant differences (marked with an asterisk in the picture) in lateralization of fractional anisotropy (FA) and weighted fractional anisotropy (wFA) of the path leaving from the auditory cortex (AC) to the amygdala (AM), and in lateralization of wFA in the path leaving from the inferior colliculus (IC) to the auditory cortex.
Based on earlier hypotheses $[2,5]$ we expected that an anatomical connection between the auditory system and the limbic system would exist and indeed, we found such a connection between the auditory cortex and the amygdala which also connected to the MGB. This suggests that DTI is able to detect an anatomical pathway which is part of the non-classical auditory pathway, e.g., the connection from the dorsal MGB to the limbic system (amygdala).

In order to summarize the track properties, we computed three quantities for each connection in each subject: the fractional anisotropy (FA), the weighted fractional anisotropy (wFA), and the connection strength (S). The anisotropy is a property of each voxel in the brain. It is a measure of the directionality of water diffusion in the voxel. If the water diffusion is primarily in a particular direction, the voxel is assumed to contain neural fibers that are oriented in that direction. A fiber track consists of a large number of neighboring voxels. The average FA of a track is thus assumed to be a measure of the patency of the track. We assumed that the wFA is an improved measure of this patency, as it takes the probability that a voxel is actually part of the track into account. Obviously, the wFA can only be computed when using probabilistic fiber tracking. Finally, the connection strength $\mathrm{S}$ is the fraction of samples in a seed region that actually reach the target region. A high strength $S$ is again a measure of the patency of the track. Conversely, a low strength may indicate that the seed region is connected primarily to other end points. Although these three measures (FA, wFA and S) are the result of considerable data reduction, they provide measures that allow for straightforward comparisons between subjects and subject groups.

By quantifying the tracks that pairwise connect the IC, $\mathrm{AC}$ and $\mathrm{AM}$, we were able to make comparisons between control subjects and tinnitus patients. These three ROIs were selected because they may play an important role in the mechanisms that lead to tinnitus. Tinnitus is an auditory percept that occurs in the absence of a known acoustical source outside the body. In many cases, tinnitus is presumably related to abnormal spontaneous neural activity in the brain. Such patterns may occur in cases of peripheral hearing loss, as reviewed in Eggermont and Roberts [52], apparently as a consequence of altered (often reduced) peripheral input to the central auditory system.

Despite the fact that a relation between tinnitus and peripheral hearing loss is present, it is not straightforward. For example, tinnitus may be present in the absence of any substantial hearing loss. Also, the presence of hearing loss is associated with tinnitus in only about $20 \%$ of the cases [53]. The mechanisms underlying the diffuse relation between hearing loss and tinnitus are unclear. It is possible that subtle characteristics of the functional or anatomical (structural) connectivity of the central auditory systems determine whether a subject develops tinnitus. In addition, nonauditory brain areas are believed to be involved in tinnitus. Specifically, the interaction between the limbic and the auditory system has been proposed in models that explain tinnitus $[5,19,54]$.

Abnormal spontaneous brain rhythms in tinnitus patients are indicative of abnormal functional connectivity in such patients. These brain rhythms reflect the activity of forward 

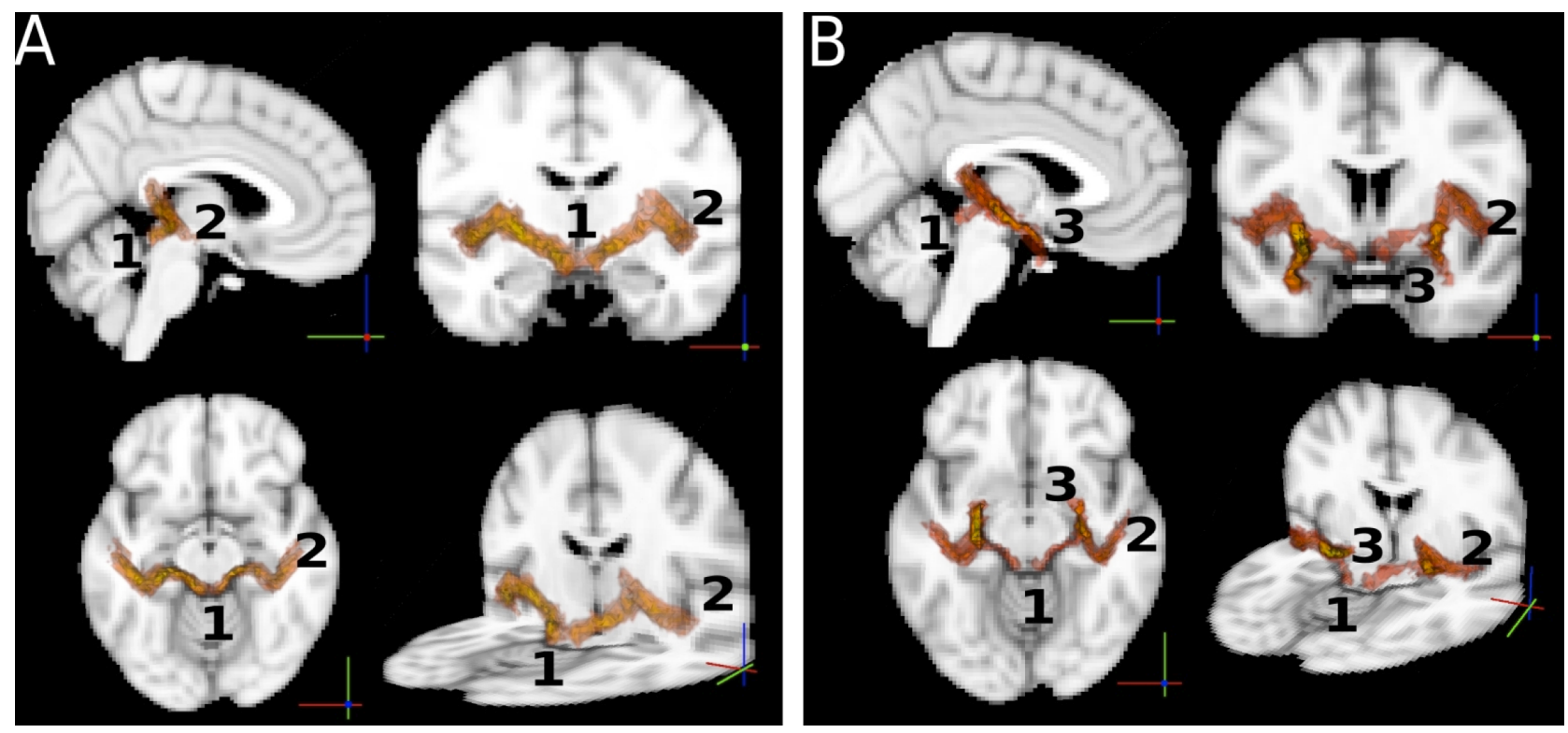

Fig. (4). A: Connections of the auditory pathways. Each path shown is the group average of the normalized (to the MNI standard template) paths of all the subjects. The intersection of two paths $(\mathrm{A} \rightarrow \mathrm{B}, \mathrm{B} \rightarrow \mathrm{A})$ between any pair $(\mathrm{A}, \mathrm{B})$ of ROIs was used to define the location of a connection. The image shows iso-probability surfaces, coded with color (the color scale ranges from yellow to red, where yellow indicates the highest probability to find the path in that brain region, and red indicates lower probabilities). Track endpoints are identified by numbers. 1: inferior colliculus, 2: auditory cortex, 3: amygdala. The iso-probability surfaces are semitransparent so that an exploration of all voxels is possible. B: the connections between the amygdala and the inferior colliculus, overlapped with the connections between the amygdala and the auditory cortex.

and backward loops connecting brain areas, specifically of the cortical-thalamical connections [55]. In tinnitus patients, the alpha brain rhythm is reduced, while the delta rhythm is substantially enhanced [56]. These abnormal brain rhythms, which differentiate tinnitus patients from control subjects, could in part be due to differences in the anatomical connections.

Our study is an attempt to show possible anatomical differences between subject groups using DTI. The computation of FA, wFA and $\mathrm{S}$ allowed us to compute such differences. We found differences and similarities between tinnitus patients and healthy controls. For example, the variability across subjects for FA and wFA of the paths was remarkably small within each group, and was also very similar between both groups.

Significant differences in path strength between tinnitus patients and healthy controls were found for the left IC-AM connection, the right AC-IC connection, and the AC-AM connection for both hemispheres (see Table 1a) and Fig. (2c), which also resulted in a significant difference for the lateralization (see Table 1b) and Fig. (3c). Tinnitus patients also showed a higher FA in the AM-AC connection.

Regarding lateralization, differences between tinnitus patients and controls were found for the FA of the AC-AM connection and the weighted FA of the AC-AM and IC-AC connections, cf. Fig. (3b). This result may correspond to the abnormal lateralization in brain function observed in tinnitus patients in a PET study [57].

The difference in strength of the connection between auditory cortex and amygdala in subjects with tinnitus compared to controls indicates that the limbic system may indeed play a major role in tinnitus, especially concerning the emotional content of the percept of tinnitus. Although cognitive therapies, focused on treating tinnitus by habitation, have been used for many years $[6,7]$, no imaging study prior to the present one has shown a potential anatomical pathway that might function differently between tinnitus patients and normal hearing controls.

\section{ACKNOWLEDGEMENTS}

This research was funded by the University of Groningen $(\mathrm{AC}, \mathrm{JR})$ and the University Medical Center Groningen, the Heinsius Houbolt Foundation and the Netherlands Organization for Scientific Research (CL, PvD).

\section{REFERENCES}

[1] Ehret G, Romand R. The Central Auditory System. Oxford: Oxford University Press 1997

[2] Møller AR, Møller MB, Yokota M. Some forms of tinnitus may involve the extralemniscal auditory pathway. Laryngoscope 1992; 102(10): 1165-71.

[3] Dalgleish T. The emotional brain. Nat Rev Neurosci 2004; 5(7): 583-9.

[4] Morgane PJ, Mokler DJ. The limbic brain: continuing resolution. Neurosci Biobehav Rev 2006; 30(2): 119-25.

[5] Jastreboff PJ. Phantom auditory perception (tinnitus): mechanisms of generation and perception. Neurosci Res 1990; 8(4): 221-54.

[6] Jastreboff PJ, Jastreboff MM. Tinnitus retraining therapy for patients with tinnitus and decreased sound tolerance. Otoralingol Clin North Am 2003; 36(2): 321-36.

[7] Jastreboff PJ. Tinnitus retraining therapy. Prog Brian Res 2007; 166: 415-23 
[8] Lanting CP, de Kleine E, van Dijk P. Neural activity underlying tinnitus generation: results from PET and fMRI. Hear Res 2009; 255(1-2): 1-13.

[9] Smits M, Kovacs S, de Ritter D, Peeters RR, van Hecke P, Sunaert S. Lateralization of functional magnetic resonance imaging (fMRI) activation in the auditory pathway of patients with lateralized tinnitus. Neuroradiology 2007; 49(8): 669-79.

[10] Melcher JR, Sigalovsky IS, Guinan JJJ, Levine RA. Lateralized tinnitus studied with functional magnetic resonance imaging: abnormal inferior colliculus activation. J Neurophysiol 2000; 83(2): 1058-72.

[11] Devlin JT, Raley J, Tunbridge E, et al. Functional asymmetry for auditory processing in human primary auditory cortex. J Neurosci 2003; 23(37): 11516-22.

[12] Basser PJ, Mattiello J, Bihan DL. Estimation of the effective selfdiffusion tensor from the NMR spin-echo. J Magn Reson 1994; 103(3): 247-54

[13] Pierpaoli C, Jezzard P, Basser PJ, Barnett A, DiChiro G. Diffusion tensor MR imaging of the human brain. Radiology 1996; 201(3): 637-48.

[14] Werring D, Clark C, Parker G, Thmopson A, Miller D. Diffusion tensor imaging of lesions and normal-appearing white matter in multiple sclerosis. Neurology 1999; 52(8): 1626-32.

[15] Werring D, Toosy A, Clark C, et al. Diffusion tensor imaging can detect and quantify corticospinal tract degeneration after stroke. J Neurosurg Psychiatry 2000; 69: 269-72.

[16] Beaulieu C. The basis of anisotropic water diffusion in the nervous system - a technical review. Nucl Magn Res Biomed 2002; 15(7-8): 435-55.

[17] Kindlmann G. Superquadric Tensor Glyphs. In IEEE TVCG/EG Symposium on Visualization 2004; pp. 147-54.

[18] Basser PJ, Pajevic S, Pierpaoli C, Duda J, Aldroubi A. In vivo fiber tractography using DT-MRI data. Magn Reson Med 2000; 44(4): 625-32.

[19] Lockwood AH, Salvi RJ, Coad ML, Towsley ML, Wack DS, Murphy BW. The functional neuroanatomy of tinnitus: evidence for limbic system links and neural plasticity. Neurology 1998; 50(1): 114-20

[20] Lanting C, Kleine ED, Bartels H, Dijk PV. Functional imaging of unilateral tinnitus using fMRI. Acta Otolaryngol 2008; 128(4): 415-21.

[21] Yoo DS, Choi WY, Lee SY, et al. Quantitative analysis of white matter on DTI images of patients with tinnitus: preliminary report. In Conf Proc IEEE Eng Med Biol Soc 2006; vol.1: pp. 1870-72.

[22] Lutz J, Hemminger F, Stahl R, et al. Evidence of subcortical and cortical aging of the acoustic pathway: a Diffusion Tensor Imaging (DTI) study. Acad Radiol 2007; 14(6): 629-700.

[23] Lee YJ, Bae SJ, Lee SH, et al. Evaluation of white matter structures in patients with tinnitus using diffusion tensor imaging. $\mathbf{J}$ Clin Neurosci 2007; 14(2): 515-19.

[24] Schmahmann JD, Pandya DN. Fiber pathways of the brain. Oxford: Oxford University Press 2006.

[25] Martin JH. Neuroanatomy: text and atlas. USA: McGraw-Hill Medical 2003

[26] Behrens T, Johansen-Berg H, Jabdi S, Rushworth MF, Woolrich MW. Probabilistic diffusion tractography with multiple fibre orientations: What can we gain? Neuroimage 2007; 34(1): 144-55.

[27] Lee SH, Chang Y, Lee JE, Cho JH. The comparison of brain functional Imaging between conductive and sensorineural hearing loss. In: Proceeding vary of Middle Ear Mechanics in Research and Otology 2003; pp. 253-59.

[28] Groof GD, Verhoye M, Meir VV, Tindemans I, Leemans A, der Linden AV. In vivo diffusion tensor imaging (DTI) of brain subdivisions and vocal pathways in songbirds. Neuroimage 2006; 29(3): 754-63.

[29] Upadhyay J, Ducros M, Knaus TA, et al. Function and Connectivity in Human Primary Auditory Cortex: A Combined fMRI and DTI Study at 3 Tesla. Cereb Cortex 2006; 17(10): 242032.

[30] Ashburner J, Friston K. Voxel-based morphometry - the methods. Neuroimage 2000; 11(6): 805-21.

[31] Jones D, Symms M, Cercignani M, Howard R. The effect of the filter size on VBM analyses of DT-MRI data. Neuroimage 2005; 26(2): 546-54.
[32] Park HJ, Kubicki M, Shenton M, et al. Spatial normalization of diffusion tensor MRI using multiple channels. Neuroimage 2003; 20(4): 1995-2009.

[33] Smith SM, Jenkinson M, Johansen-Berg H, et al. Tract-based spatial statistics: voxelwise analysis of multi-subject diffusion data. Neuroimage 2006; 31(4): 1487-505.

[34] Hall DA, Haggard MP, Akeroyd MA, et al. Sparse temporal sampling in auditory fMRI. Human Brain Mapp 1999; 7(3): 21323

[35] Deichmann R, Josephs O, Hutton C, Corfield DR, Turner R. Compensation of susceptibility induced BOLD sensitivity losses in echo planar fMRI imaging. Neuroimage 2002; 15(1): 120-35.

[36] Baumgart F, Kaulisch T, Tempelmann C, et al. Electrodynamic headphones and woofers for application in magnetic resonance imaging scanners. Med Phys 1998; 25(10): 2068-70.

[37] Langers DRM, Backes WH, van Dijk P. Spectrotemporal features of the auditory cortex: the activation in response to dynamic ripples. Neuroimage 2003; 20(1): 265-75.

[38] Wang R, Wedeen JV, Org T. Martinos Center for Biomedical Imaging, Massachusetts General Hospital, 2007; Available at http: //www.trac-kvis.org

[39] Smith SM, Jenkinson M, Woolrich MW, et al. Advances in functional and structural MR image analysis and implementation as FSL. Neuroimage 2004; 23: 208-19.

[40] Statistical Parametric Mapping 5, 2005; available at http //www.fil.ion.ucl.-ac.uk/spm/

[41] Jezzard P, Balaban RS. Correction for geometric distortions in echoplanar images from B0 field variations. Magn Reson Med 2005; 34(1): 66-73.

[42] Andersson JLR, Skare S, Ashburner J. How to correct susceptibility distortions in spin-echo echo-planar images: application to diffusion tensor imaging. Neuroimage 2003; 20(2) 870-88

[43] Farrell JA, Landman BA, Jones CK, et al. Effects of signal-to-noise ratio on the accuracy and reproducibility of diffusion tensor imaging-derived fractional anisotropy, mean diffusivity, and principal eigenvector measure-ments at $1.5 \mathrm{~T}$. J Magn Reson Imaging 2007; 26(3): 756-67.

[44] Smith SM. Fast robust automated brain extraction. Hum Brain Map 2002; 17(3): 143-55

[45] Brett M, Johnsrude IS, Owen AM. The problem of functional localization in the human brain. Nat Rev Neurosci 2002; 3: 243-49.

[46] Morosan P, Rademacher J, Schleicher A, Amunts K, Schormann T, Zilles K. Human primary auditory cortex: Cytoarchitectonic subdivisions and mapping into a spatial reference system. Neuroimage 2001; 13(4): 684-701.

[47] Amunts K, Kedo O, Kindler M, et al. Cytoarchitectonic mapping of the human amygdala, hippocampal region and entorhinal cortex: intersubject variability and probability maps. Anat Embryol (Berl) 2005; 210(5-6): 343-52.

[48] Anwander A, Tittgemeyer M, von Cramon DY, Friederici AD, Knösche TR. Connectivity-Based Parcellation of Broca's area. Cereb Cortex 2007; 17(4): 816-25.

[49] Behrens TEJ, Woolrich MW, Jenkinson M, et al. Characterization and propagation of uncertainty in diffusion-weighted MR imaging. Magn Reson Med 2003; 50(5): 1077-88.

[50] Jenkinson M, Smith S. A global optimisation method for robust affine registration of brain images. Med Image Anal 2001; 5(2): 143-56.

[51] Dauguet J, Berezovskii SPV, Delzescaux T, Born SKWR, Westin CF. Comparison of fiber tracts derived from in-vivo DTI tractography with $3 \mathrm{D}$ histological neural tract tracer reconstruction on a macaque brain. NeuroImage 2007; 37(2): 530-38.

[52] Eggermont JJ, Roberts LE. The neuroscience of tinnitus. Trends Neurosci 2004; 27(11): 767-82.

[53] Lockwood AH, Salvi RJ, Burkard RF. Tinnitus. N Engl J Med 2002; 347(12): 904-10.

[54] Møller AR. Hearing. Anatomy, Physiology, and Disorders of the Auditory System 2nd ed. London, Academic Press 2006.

[55] Llinas R, Urbano FJ, Leznik E, Ramirez RR, vanMarle HJ. Rhythmic and dysrhythmic thalamocortical dynamics: GABA systems and the edge effect. Trends Neurosci 2005; 28(6): 325-33.

[56] Weisz N, Moratti S, Meinzer M, Dohrmann K, Elbert T. Tinnitus perception and distress is related to abnormal spontaneous brain 
activity as measured by magnetoencephalography. PLoS Med 2005; 2(6): 546-53.

[57] Langguth B, Eichhammer P, Kreutzer A, et al. The impact of auditory cortex activity on characterizing and treating patients with chronic tinnitus--first results from a PET study. Acta Otolaryngol Suppl 2006; 556: 84-8.

Received: May 13, 2009

Revised: June 01, 2009

Accepted: October 13, 2009

(c) Crippa et al.; Licensee Bentham Open.

This is an open access article licensed under the terms of the Creative Commons Attribution Non-Commercial License (http://creativecommons.org/licenses/by-nc/3.0/) which permits unrestricted, non-commercial use, distribution and reproduction in any medium, provided the work is properly cited. 